\title{
Shot noise variation within ensembles of gold atomic break junctions at room temperature
}

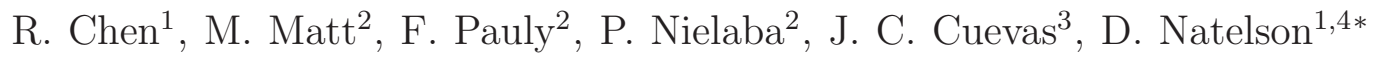 \\ 1 Department of Physics and Astronomy, Rice University, \\ 6100 Main St., Houston, TX 7r005, USA \\ 2 Department of Physics, University of Konstanz, D-78457 Konstanz, Germany \\ 3 Departamento de Física Teórica de la Materia Condensada \\ and Condensed Matter Physics Center (IFIMAC), \\ Universidad Autónoma de Madrid, E-28049 Madrid, Spain and \\ 4 Department of Electrical and Computer Engineering, \\ Rice University, 6100 Main St., Houston, TX 7r005, USA
}

\begin{abstract}
Atomic-scale junctions are a powerful tool to study quantum transport, and are frequently examined through the mechanically controllable break junction technique (MCBJ). The junctionto-junction variation of atomic configurations often leads to a statistical approach, with ensembleaveraged properties providing access to the relevant physics. However, the full ensemble contains considerable additional information. We report a new analysis of shot noise over entire ensembles of junction configurations using scanning tunneling microscope (STM)-style gold break junctions at room temperature in ambient conditions, and compare this data with simulations based on molecular dynamics (MD), a sophisticated tight-binding model, and nonequilibrium Green's functions. The experimental data show a suppression in the variation of the noise near conductances dominated by fully transmitting channels, and a surprising participation of multiple channels in the nominal tunneling regime. Comparison with the simulations, which agree well with published work at low temperatures and ultrahigh vacuum (UHV) conditions, suggests that these effects likely result from surface contamination and disorder in the electrodes. We propose additional experiments that can distinguish the relative contributions of these factors.
\end{abstract}

\footnotetext{
* natelson@rice.edu corresponding author
} 


\section{INTRODUCTION}

Out of both fundamental and technological motivation, atomic-scale junctions between conductors have been studied extensively in recent years [1]. The size of the "active" region of such devices is smaller than the inelastic mean free path for electrons, and only a few quantum channels contribute to conduction; thus such junctions serve as a simplified platform to study quantum transport. By inserting molecules between such electrodes, similar physics studies have been extended to molecular junctions. The MCBJ technique enables study of these junctions as a function of electrode separation, with each junction configuration sampling from an ensemble of atomic arrangements. The varying mechanical and electrical properties of junctions can be examined, enabling studies of conductance quantization [2, 3], energy dissipation in atomic ballistic wires [4], electron-phonon interaction modifications to the shot noise [5, 6], flicker noise in metal junctions [7], Joule heating in the electrodes [8], and so on.

In most of these works, statistical analyses are usually conducted to average out the microscopic variability of junction configurations. For instance, conductance histograms [9 11$]$ are a common tool to study the preferred conductance values, and hence preferred sets of discrete quantum channel transmittances, averaged over accessible atomic configurations. The obvious shortcoming is that information associated with each specific atomic arrangement is lost in the averaging.

To go beyond this ensemble-averaged analysis, considerable efforts have been made in recent years. Measurements of multiple Andreev reflection reveal the transmittance of each specific quantum channel in individual superconductor junctions [3, 12]. From ensembles, the range of mathematically allowed transmittances may be estimated [13]. In the mean time, the development of new analysis techniques also enables more information to be extracted from the mountain of data over all the ensembles. These methods include "density plots", where every experimental data point in the ensemble is counted. Examples are density plots of conductance vs. elongation [14 16], current-voltage characteristics [17-19], and mechanical stiffness vs. elongation [20]. Two-dimensional (2D) cross-correlation methods also take advantage of entire ensembles, revealing detailed information about motifs of junction formation [21 23].

Over the same time period, there have also been many advances in computational tech- 
niques, particularly the ability to combine $\mathrm{MD}$ with quantum transport to describe the inherent interplay between mechanical and electrical properties in these systems, which is crucial to establish a direct comparison with the experimental results [24 27].

In this work, we analyse conductance and shot noise measurements over whole ensembles of STM-style gold break junctions at room temperature, expanding upon prior ensembleaveraged treatments [28 30]. When mapping out two dimensional density plots of shot noise vs. conductance, we find the standard deviation (over the ensemble) in the noise at each conductance, $G$. At conductances in the nominal tunneling regime $\left(G<1 G_{0} \equiv 2 e^{2} / h\right)$, we find a nonzero variance, which indicates that in our system several quantum channels frequently contribute to transport in this regime. If normalized by the average noise, the resulting fractional variance in the noise as a function of conductance is relatively featureless, while the nonzero fractional variance in the tunneling region still survives. Furthermore, the standard deviation of the shot noise shows clear minima coincident with the ensemble-averaged shot noise spectral density suppressions and conductance peaks near integer multiples of $G_{0}$. A state-of-the-art calculation combining MD, an accurate tight-binding model, and Green's function techniques to examine junction formation is compared to these experimental results. As we discuss below, surface contamination and disorder in the electrodes are likely responsible for many of the differences between the experiment and the calculations.

Shot noise, firstly discussed by Schottky in the context of vacuum diodes [31], originates from the discreteness of charge carriers [32]. This nonequilibrium noise only exists in addition to the Johnson-Nyquist noise [33, 34] at a finite bias. For Poisson-distributed, uncorrelated electrons, the shot noise spectral density $\left(\mathrm{A}^{2} / \mathrm{Hz}\right)$ is $S_{I}=2 e I$, where $e$ is the electronic charge and $I$ is the average current. Correlations between the electrons modify the noise, which is often written in the form $S_{I}=2 e I F$, where $F$ is the Fano factor. Measurements of $F$ can therefore provide insight into the interactions of the electrons with each other [35, 36] and other degrees of freedom such as phonon modes [5, 6].

In atomic-scale junctions the electronic transport is generally described well by the Landauer-Büttiker picture [37 39], where electron-electron interactions are neglected and electrons transport coherently. The conductance is then $G=G_{0} \sum_{i} \tau_{i}$, where $\tau_{i}$ represents the transmittance of each discrete quantum channel indexed by $i$. The associated shot noise 
at zero temperature satisfies the following form $[40,44]$

$$
S_{I}=2 e V G_{0} \sum_{i=1}^{N} \tau_{i}\left(1-\tau_{i}\right)
$$

where $V$ is the bias voltage across the junction. The corresponding Fano factor is given by $F \equiv \sum_{i=1}^{N} \tau_{i}\left(1-\tau_{i}\right) / \sum_{i=1}^{N} \tau_{i}$, where $N$ is the number of open conduction channels. At elevated temperatures shot noise is enhanced and becomes entwined with Johnson-Nyquist noise. The total spectral density of the current noise is then

$$
S_{I}=G_{0}\left[4 k_{\mathrm{B}} T \sum_{i=1}^{N} \tau_{i}^{2}+2 e V \operatorname{coth}\left(\frac{e V}{2 k_{\mathrm{B}} T}\right) \sum_{i=1}^{N} \tau_{i}\left(1-\tau_{i}\right)\right] .
$$

This relation is relatively more complicated, but can still be expressed in the form of the transmittance set $\left\{\tau_{i}\right\}$. We define the excess noise $P \equiv S_{I}(V)-S_{I}(0)$ as the finite-

temperature shot noise. In a practical measurement, other noise sources, such as flicker noise, can also contribute to the measured noise, and must be considered case by case. When shot noise dominates the excess noise, it provides information about each quantum channel through the $\tau_{i}\left(1-\tau_{i}\right)$ term, while the conductance only reflects the overall contribution from all the channels. In an individual junction, if no more than two channels dominate conduction, shot noise and conductance measurements together allow determination of all the transport details. If more than two channels are involved, the exact transmittance values cannot be completely determined, but instead a mathematically allowed range of each transmittance may be extracted [13].

\section{EXPERIMENTAL METHODS}

The measurements are conducted across STM-style gold break junctions at room temperature in air. As described in our previous publications [29, 30], a gold tip attached to an end of one piezo actuator is electrically controlled, moving towards and away from an evaporated thick gold film, allowing the cyclic formation and breaking of gold junctions. Only the data collected during the breaking part of the cycle are analysed, with each breaking half cycle defined as one trace. At each fixed "DC" bias $V$, broad band (250 - $500 \mathrm{MHz}$ ) excess noise and DC conductance measurements are performed simultaneously using a lock-in technique. At the radio frequency bandwidth, the dominant contributor of excess noise is shot noise, as discussed in detail elsewhere [30]. The gold tip is controlled to obtain approximately one 
trace per second. Conductance and noise power from lock-in outputs are acquired at $10^{5}$ samples/s, but with the time constant on each lock-in set to $100 \mu \mathrm{s}$. In each trace, the time-averaged (with sub-millisecond averaging periods) shot noise power as a function of conductance is computed. Both noise power and conductance axes are binned. The junction conductance is calculated from the measured current and applied voltage bias, taking into account series resistances in the measurement circuit. The statistical uncertainty in the conductance data is less than $1 \%$. The statistical uncertainty in the measurement of the rf power is at a similar level, though the background contributes a systematic uncertainty to individual measurements. The number of counts in each bin $(G, P)$ is used to construct a $2 \mathrm{D}$ trace density plot.

Typical traces have from five to more than ten thousand counts between $G=4 G_{0}$ and $G=0.01 G_{0}$. Traces with less than 1000 counts are not included as these indicate anomalously rapid breakage or a measurement problem; varying this cutoff from 500 counts to 2000 counts produces no noticeable change in the resulting plots or analysis. For clarity of color scale when plotting the trace density, at each conductance the bins are normalized to show the relative probability (between 0 and 1) of measuring a particular noise power.

In the analysis, a subtle technical issue arises regarding the rigorous extraction of the shot noise from raw data. The noise power detector has small random fluctuations in addition to the true target signal, causing a small but non-negligible positive background in the lock-in amplifier measurement of its amplitude. When performing a full ensemble average analysis, statistical methods [29] can be applied to remove this small background, as we have done previously. However, this background removal method does not work rigorously at the single trace level; thus, in this work this background remains. Under this circumstance, there is therefore a slight systematic overestimate of the true shot noise spectral density, especially

relevant when shot noise is small. The background is independent of $G$ and is equivalent to approximately $0.1 \times 10^{-24} \mathrm{~A}^{2} / \mathrm{Hz}$, and sets the "floor" of the data shown in Fig. 1, panels (c) and (d).

\section{EXPERIMENTAL RESULTS}

Figure 1 shows the resulting density plots obtained at biases of $180 \mathrm{mV}$ and $120 \mathrm{mV}$, respectively. The top-most panels are the conductance histograms, commonly used to find the 

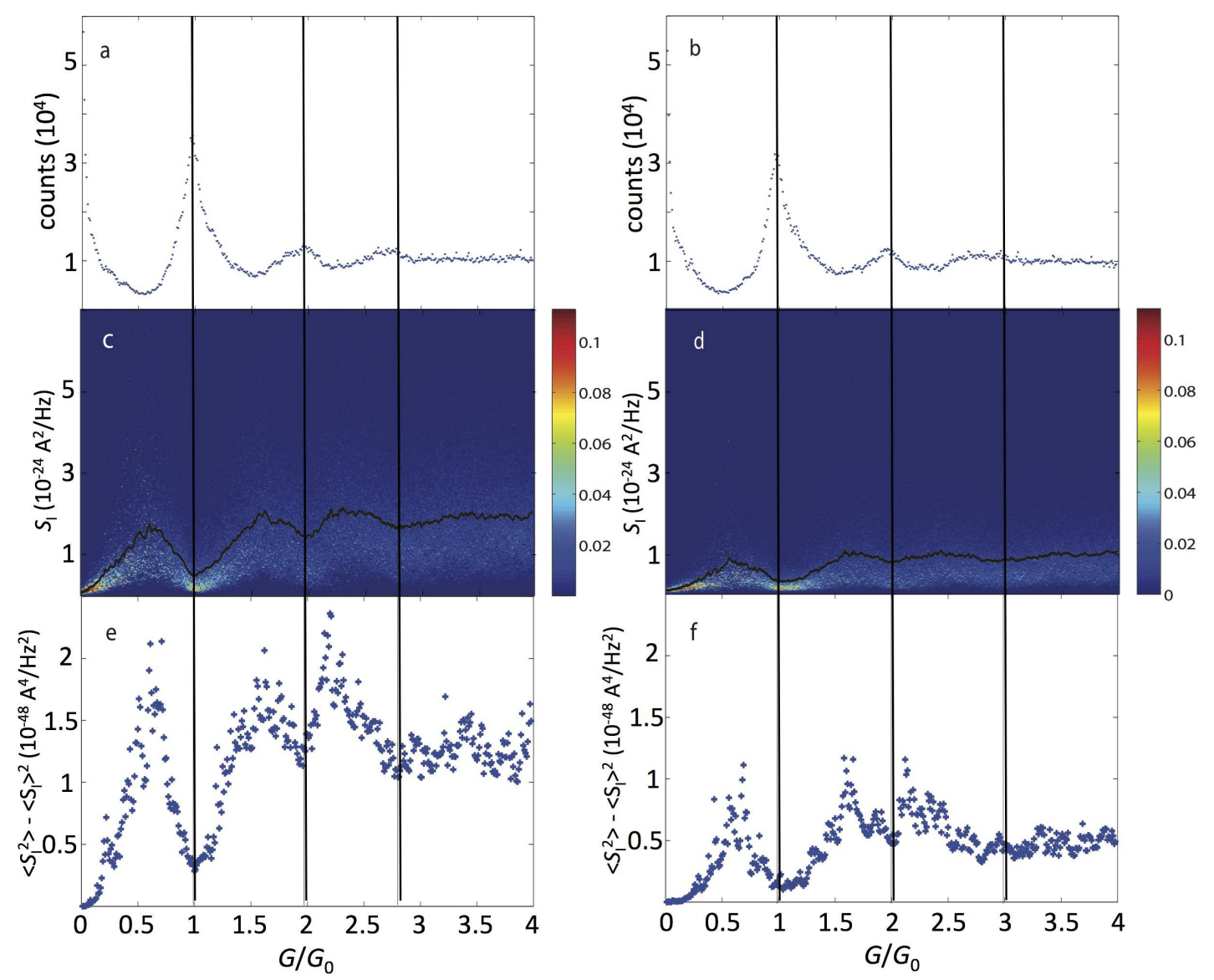

FIG. 1. Noise and its variation across ensembles. (a) Conductance histogram acquired with 180 $\mathrm{mV}$ bias (816 traces). (b) Conductance histogram acquired with $120 \mathrm{mV}$ bias (807 traces). (c, d) $2 \mathrm{D}$ density plots for the respective ensembles, with data at each conductance normalized to show the probability for finding a particular shot noise power value at any particular conductance. The black curves show the ensemble-averaged noise power. (e, f) The associated variance of shot noise at each conductance. The vertical black lines are guides to the eye to indicate the conductance for which the shot noise variance is a minimum.

preferred conductance values. The middle panels are the trace density plots, where warmer colors indicate higher densities of traces. The black curve indicates the ensemble-averaged excess noise power as a function of conductance. From this panel, clearly the distribution of shot noise has some interesting features. The "envelope" of the traces is comparatively large away from the conductances where shot noise is suppressed, while that envelope becomes significantly concentrated at those suppression regions near integer multiples of $G_{0}$.

To quantify this distribution, the variance $\left\langle S_{I}^{2}\right\rangle-\left\langle S_{I}\right\rangle^{2}$ at each conductance is computed 
and shown in panels $(e, f)$. Note that the shot noise variance minima are coincident with the conductance peaks and noise suppressions. Ensembles acquired at many other biases, or with a different radio frequency bandwidth, reproduce the same features. According to equations (1) and (2), shot noise suppressions originate from the fully transmitting channels. It is also widely known that shot noise suppressions in gold junctions coincide with the conductance peaks [45], indicating a potential relation between the fully transmitted channels and one or a few preferred atomic arrangements existing at the conductance plateaus. There is no obvious theoretical explanation for these shot noise variance minima. We infer that somehow $\left\{\tau_{i}\right\}$ has limited variations at these preferred conductance values compared to higher or lower conductances. Either relatively fewer atomic configurations are allowed at the conductance plateaus, or the allowed atomic configurations have relatively similar transmittance sets $\left\{\tau_{i}\right\}$, or both.

The density plots also reveal information about the nature of the shot noise suppression at the conductances indicated by the peaks in the conductance histograms. At cryogenic temperatures, theory and experiments agree very well that shot noise in gold junctions is suppressed down to near zero at conductance plateaus [46, 47], particularly when $G \approx G_{0}$. This indicates a single fully transmitted channel at $1 G_{0}$ and some limited channel mixture at the higher $G$ plateaus. In our ensemble-averaged measurements, all the suppressions are only partial [29]. The density plot reveals the explanation: At $1 G_{0}$, most traces do show nearly complete suppression, while a small fraction relatively elevated noise. At other plateaus, though the lower count numbers and sparse distributions make the situation less obvious, the most populated shot noise values are also lower than the ensemble average. These observations show that the channel mixing responsible for affecting the depth of the noise suppression takes place only in a subset of the full ensemble. This is consistent with the channel mixing resulting from either particular realizations of disorder in the electrodes or surface contamination. The peaks in the conductance histograms are consistent with deviations from integer quantization in Au junctions previously ascribed to work hardening (and therefore disorder in the electrodes) [10].

The nonzero shot noise variance in the nominal tunneling regime also indicates a breakdown of the single-channel picture below $1 G_{0}$ in these ambient condition experiments. In single channel transport, the summation in equations $(1)$ or $(2)$ reduces to $\tau(1-\tau)$ while total conductance becomes proportional to $\tau$ of the only channel. The resulting Fano factor 
is $1-\tau$, and at a given $G$ its variance is zero. Thus a nonzero noise variance when $G<G_{0}$ clearly indicates the participation of multiple quantum channels. The diffusive-like features at high conductance reported in our former paper also indicate that the channel mixture in the high conductance region in our measurements is stronger than commonly expected [29].

A debatable question here is whether the variance $\left\langle S_{I}^{2}\right\rangle-\left\langle S_{I}\right\rangle^{2}$ is a fair way to examine shot noise's variation over ensembles. In figure 2 we plot the fractional variance $\left(\left\langle S_{I}^{2}\right\rangle-\right.$ $\left.\left\langle S_{I}\right\rangle^{2}\right) /\left\langle S_{I}\right\rangle^{2}$, using the same data as in figure 1. After this normalization, the fractional variance as a function of conductance tends to be relatively featureless or even have maxima at the preferred conductances. This suggests that the variance in the noise is approximately proportional to the square of the noise itself, though there is no obvious explanation for this. The nonzero noise variance below $1 G_{0}$ naturally remains.
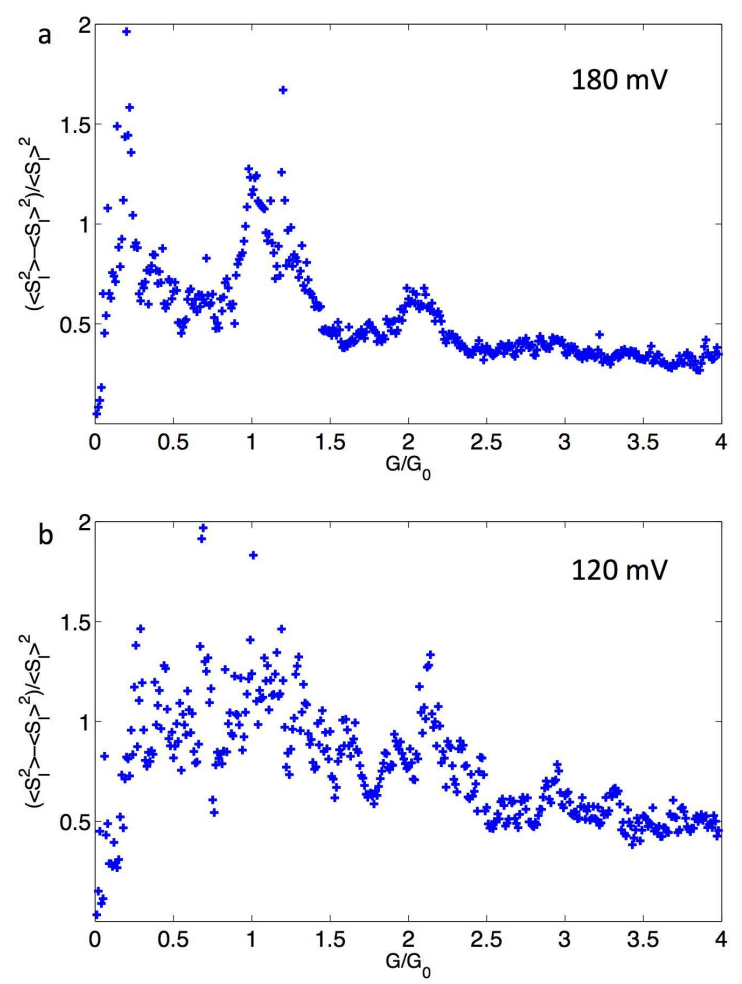

FIG. 2. Fractional variance of the noise power as a function of conductance, using the same data as in figure 1 . 


\section{SIMULATION METHODS}

This section is devoted to the description of the theoretical methods that we employed to shed some light on our experimental results for the shot noise of Au atomic-size contacts. For this purpose, we combined classical MD simulations of the formation of atomic-size contacts with a tight-binding description of the electronic structure to compute the transport properties with the help of Green's function techniques. Our approach follows closely Refs. [24, 25, 27, 48], and we now proceed to explain it in some detail.

In these atomic wires there is an intricate relation between the mechanical and the transport properties. For this reason, and in order to establish a direct comparison with our experiments, it is necessary to describe the formation process of these atomic-size contacts. For this purpose, we carried out classical MD simulations using the open source program package LAMMPS [49, 50]. These simulations are based on the so-called embedded atom method and, in particular, we employed the empirical potentials from Ref. [51]. Let us emphasize that these potentials overcome several problems of two-body potentials like, for instance, the coordination independence of the bond strength. This is important for our calculations because in our atomic contacts we have, in particular, regions with low coordination number. To generate the geometrical configurations, we start with an ideal face-centered cubic lattice, where the crystal direction $\langle 100\rangle$ lies parallel to the $z$ axis, which corresponds to the transport and elongation direction. For the MD calculations, we divided the wire geometry into three parts: Two electrodes connected by a central wire (see figure 31). The electrodes consist of 661 atoms each and they are kept fixed during the MD calculations. On the other hand, the wire is made up of 563 atoms which follow the Newtonian equations of motion. We assume a canonical ensemble and use the velocity Verlet integration scheme [52]. The wire has an initial length of $0.83 \mathrm{~nm}$ and the starting velocities of the atoms in the wire were chosen randomly with a Gaussian distribution to yield the desired average temperature. As we discuss below, we performed simulations at room temperature $(T=300 \mathrm{~K})$, but also at cryogenic temperatures $(T=4 \mathrm{~K})$ to compare with previously published results. Because of the randomness in the initial velocities, every elongation process evolves differently, while a Nose-Hoover thermostat keeps the temperature fixed [52]. To relax the system, the wire was equilibrated for 0.1 ns. Finally, the elongation process is simulated by separating the electrodes at a constant velocity of $0.4 \mathrm{~m} / \mathrm{s}$. During this process, every $10 \mathrm{ps}$ the geometry 


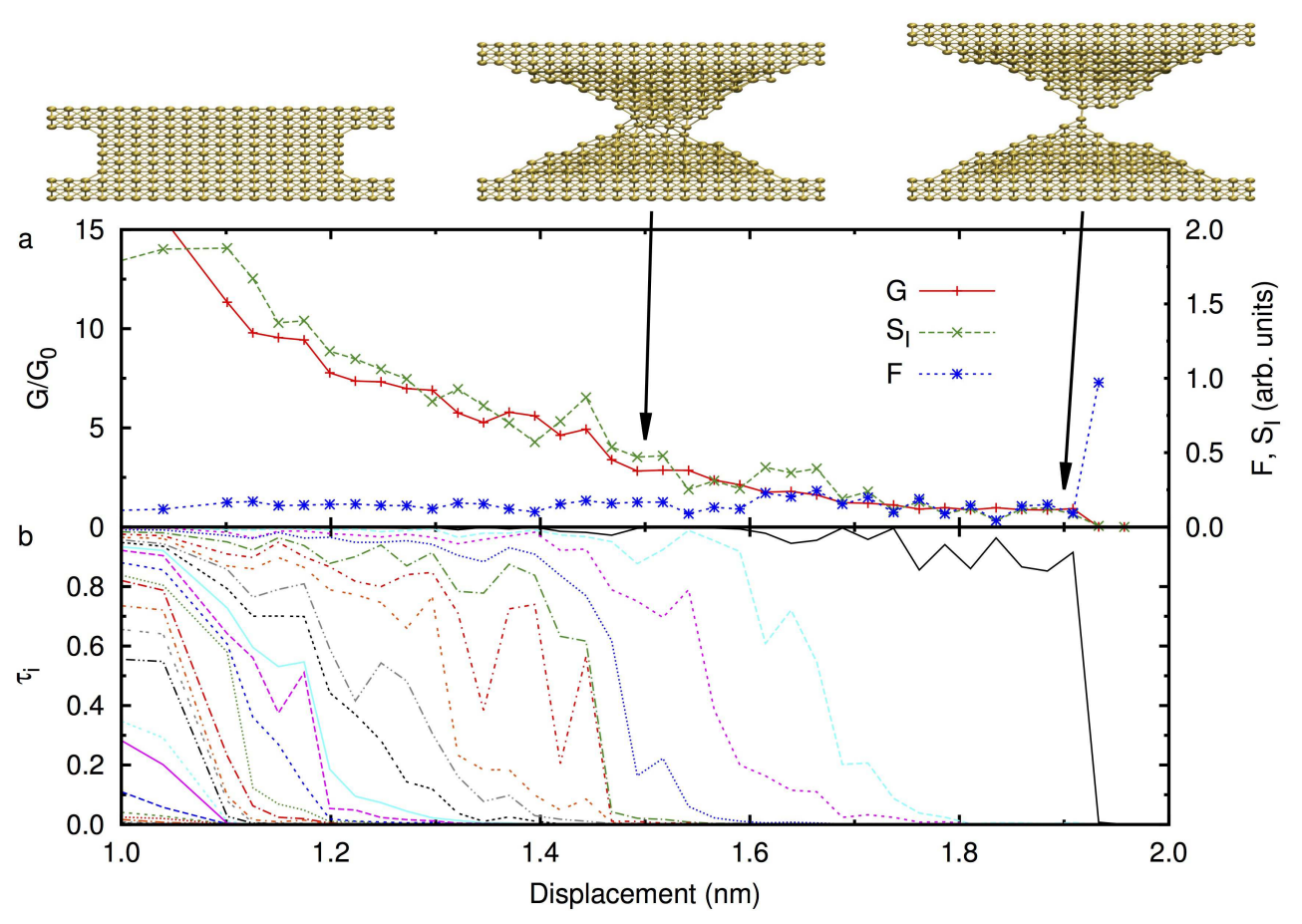

FIG. 3. Example of our simulations of the stretching of a Au wire at $300 \mathrm{~K}$. (a) Conductance, shot noise, and Fano factor as a function of the elongation of the wire. (b) Individual transmission coefficients as a function of the elongation. The upper panels show the wire geometries at different stages of the elongation process. The left geometry corresponds to the initial configuration of the simulated wire.

is recorded. A stretching process needs a total simulation time of about $4.5 \mathrm{~ns}$, until the contact breaks.

Once the geometries of the atomic wires were determined through the MD simulations, we used them to compute the conductance and the shot noise in the spirit of the LandauerBüttiker formalism. As explained in the introduction, within this formalism the transport properties are fully determined by the set of transmission coefficients $\left\{\tau_{i}\right\}$. We computed these coefficients by combining an appropriate description of the electronic structure of the $\mathrm{Au}$ wires with non-equilibrium Green's function techniques following a standard recipe that we have explained in detail elsewhere [24, 39, 53]. The electronic structure was described within the framework of the Slater-Koster tight-binding parameterization of Ref. [54], which has been quite successful in determining a variety of properties of these atomic wires [39]. To compute the transmission coefficients with the help of the Green's function techniques, 
we divided the system into three regions as in the MD simulations, i.e. the upper and lower electrodes and the central wire. The electrodes were considered to be semi-infinite perfect crystals. Their surface Green's functions were computed with the help of a decimation

technique [53, 55], using the same tight-binding parameterization as for the central part to determine their electronic structure. It is worth stressing that we enforced charge neutrality for all the atoms of the wire, which is a condition that is typically fulfilled in metallic systems [56].

Finally, to accumulate sufficient statistics for our study, we carried out $100 \mathrm{MD}$ simulations of the breaking of the Au atomic wires and checked that this number is sufficient, especially, to converge the conductance histograms. We show in figure 3 a typical example of the simulation of stretching of a wire at room temperature. In particular, we show the different physical properties of interest in the last stages of the breaking of the wire, namely the conductance, shot noise, and Fano factor. Additionally, we show the individual transmission coefficients $\tau_{i}$ as a function of the elongation.

\section{SIMULATION RESULTS AND COMPARISON WITH THE EXPERIMENTS}

Let us now discuss the main results of the simulations described in the previous section. We stress that we shall be discussing results at room temperature $(T=300 \mathrm{~K})$ to compare directly with the experimental results presented above, but we shall also present results at low temperatures $(T=4 \mathrm{~K})$ to compare with previously published experimental results obtained at cryogenic temperatures and under ultra-high vacuum conditions [13, 47].

Our main results are summarized in figure 4, where we show the conductance histograms, density plots of the shot noise, and the noise variance as a function of the conductance for 4 and $300 \mathrm{~K}$. The conductance and the noise were computed in the linear response regime, i.e. using the zero-bias transmission coefficients. Moreover, for the noise, we focus on the shot noise contribution to the excess noise by merely plotting the quantity $2 e G_{0} V \sum_{i=1}^{N} \tau_{i}\left(1-\tau_{i}\right)$ in equation (2), which ignores the contribution of the thermal noise. This is justified as $k_{\mathrm{B}} T$ is much smaller than $\mathrm{eV}$ in our experiments. For comparison with the experiments we have set the bias voltage in the prefactor to $180 \mathrm{mV}$. As one can see in panels (a) and (b), the conductance histograms are dominated by a pronounced peak at $1 G_{0}$, which is much higher at low temperatures. This is due to the fact that this peak partially originates from the 

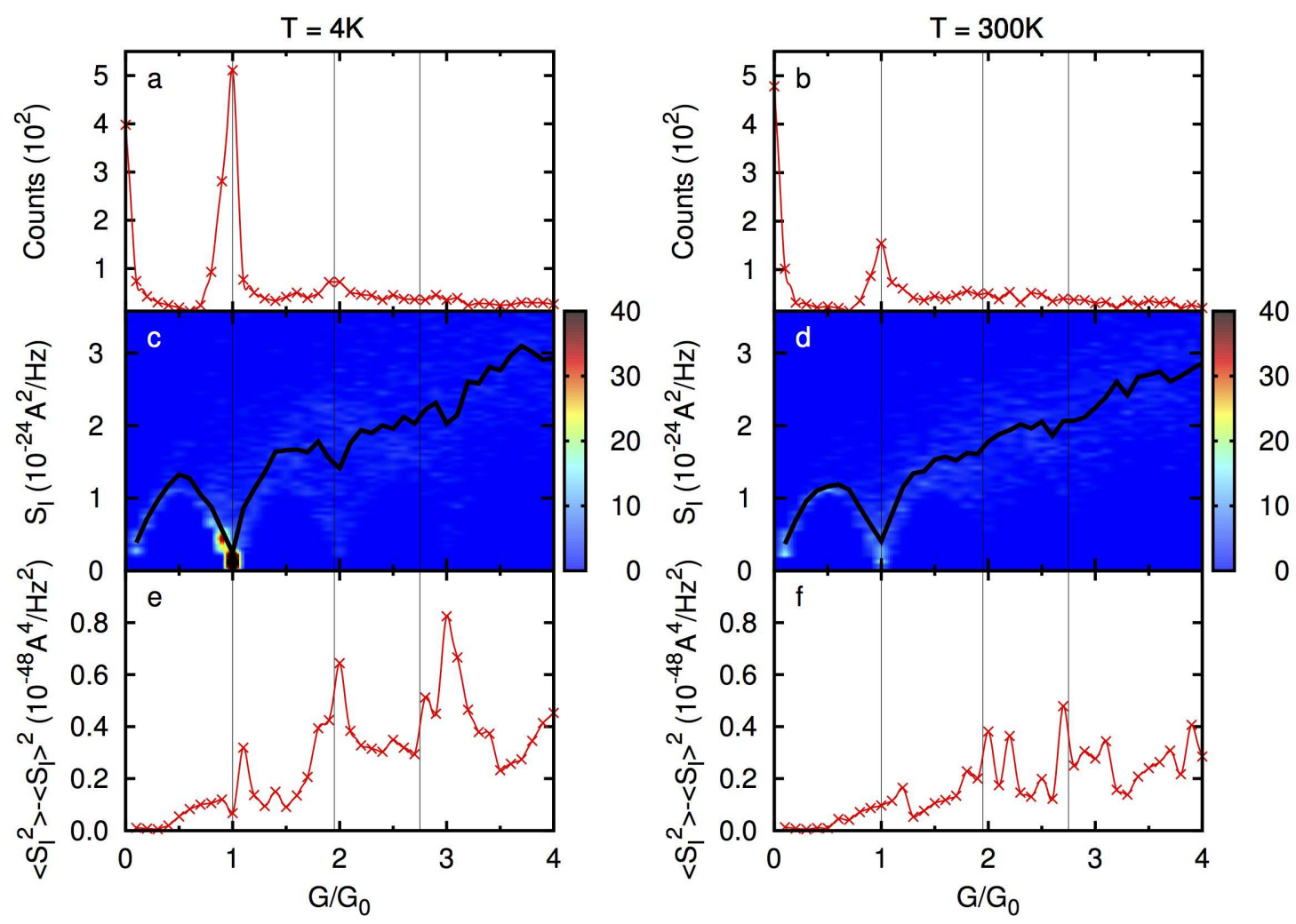

FIG. 4. (a,b) Conductance histogram obtained from 100 MD simulations at 4 and $300 \mathrm{~K}$, respectively. (c,d) The corresponding 2D density plots for the shot noise. The black solid lines indicate the ensemble-averaged values. (e,f) The associated variance of the shot noise as a function of the conductance. The bias voltage for the calculation of $S_{I}$ is set to $180 \mathrm{mV}$. The vertical lines are at the same position as in figure 1

formation of monoatomic chains [57, 58]. These chains exhibit a conductance very close to $1 G_{0}$ and we find that their formation is clearly less favorable at room temperature. Notice also the appearance of another pronounced peak at $2 G_{0}$ at low temperatures, which is to a large extent washed out at room temperature. Turning to the noise (figure $4(\mathrm{c}, \mathrm{d})$ ), the most noticeable feature is its suppression close to multiples of $G_{0}$, which is specially pronounced around $1 G_{0}$ and at $T=4 \mathrm{~K}$. This is consistent with the measurements reported above and supports the assumption that the channel mixture is stronger outside the tunneling region at higher temperatures. On the other hand, the variance of the shot noise (figure 廿(e,f)) generally exhibits maxima correlated with the maxima in the conductance histograms. While this is consistent with the low-temperature results reported by Vardimon et al. [59], this is clearly at odds with our experimental results (figure 1(e,f)). Notice also that the variance 

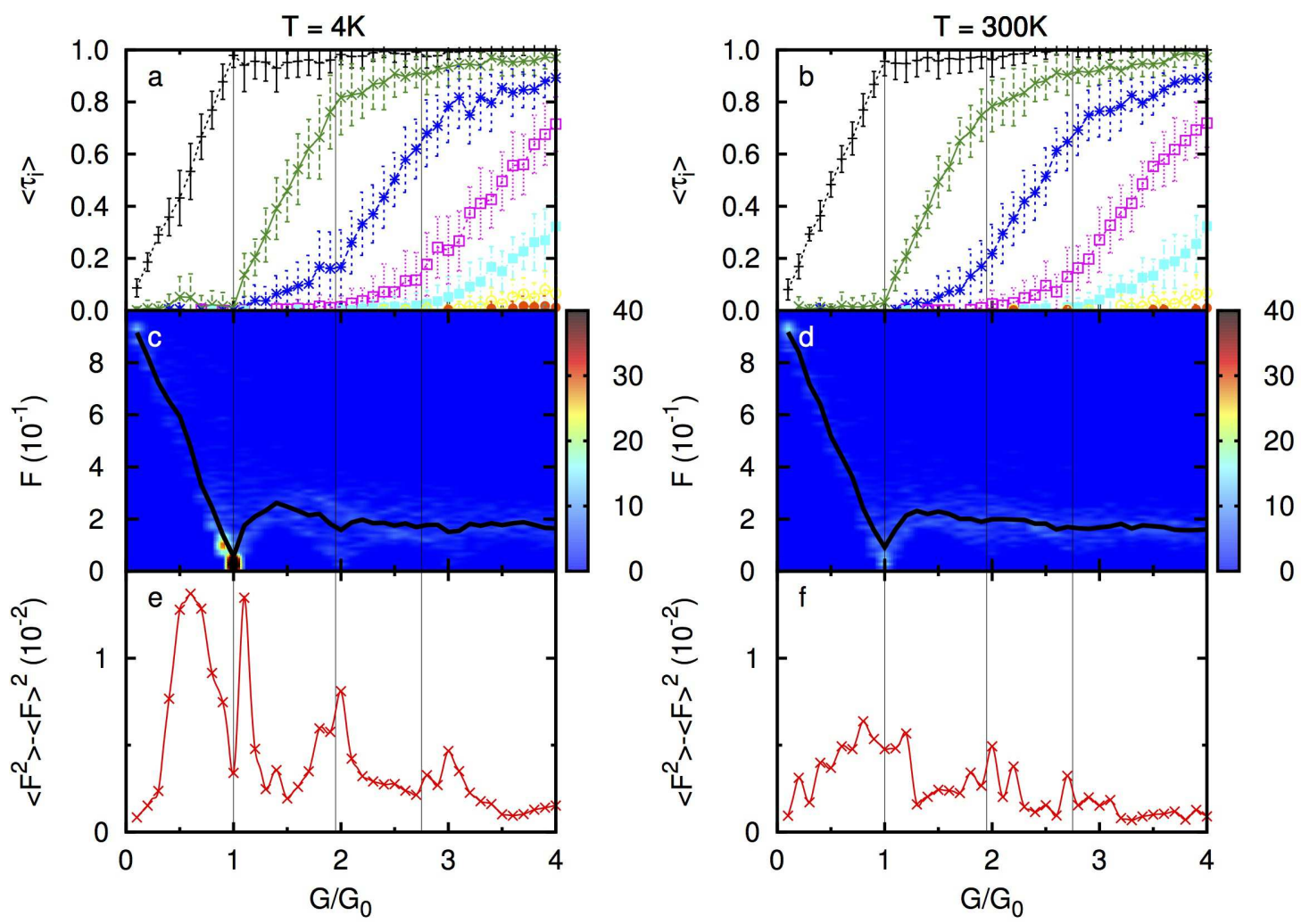

FIG. 5. (a,b) Channel distributions corresponding to the results of figure 4 at temperatures of 4 and $300 \mathrm{~K}$, respectively. (c,d) The corresponding 2D density plots for the Fano factor. The black solid lines indicate the ensemble-averaged values. $(\mathrm{e}, \mathrm{f})$ The associated variance of the Fano factor as a function of the conductance.

of the noise does not vanish below $1 G_{0}$, which clearly suggests that several channels are contributing in that region.

The origin of the results just described can be clarified by analysing both the distribution of transmission coefficients, $\left\{\tau_{i}\right\}$, and the Fano factor and its corresponding variance. This information is displayed in figure 5. The channel distributions shown in panels (a) and (b) indicate that the conduction channels open one by one as the conductance increases, as reported in Ref. [12] with the help of proximity-induced superconductivity in Au atomic-size contacts. This is a typical behavior of a monovalent metal. Focusing on the low temperature results, we find that although the conductance region $G<G_{0}$ is largely dominated by a single channel, a second one gives a sizeable contribution. This additional channel manifests as a kink in the Fano factor around $0.5 G_{0}$ and as a pronounced maximum in the Fano factor variance at that position. It is worth stressing that the presence of this second 
channel does not "spoil" the conductance quantization and the histogram still exhibits a very pronounced peak at $1 G_{0}$. For higher conductance, the Fano factor exhibits a partial suppression close to multiples of $G_{0}$, while its variance has maxima correlated with the maxima of the conductance or the minima of the shot noise. All these findings at $4 \mathrm{~K}$ are in very good agreement with the recent experimental results of Refs. [13, 59] where shot noise was measured at cryogenic temperatures and used to extract the channel distribution of $\mathrm{Au}$ few-atom contacts. Turning now to the room temperature results, one can still see a small contribution of a second channel for $G<G_{0}$, which explains the non-vanishing variance of both the shot noise and the Fano factor for these conductances. However, at 300 $\mathrm{K}$ the weight of this channel is not sufficient to produce a maximum in the variance of these quantities below $G_{0}$. For higher conductance, the variance of the Fano factor exhibits again shallow maxima correlated with the maxima of the conductance and the minima of the shot noise.

From the previous discussion, a natural question arises: What is the origin of the second channel that appears in some geometries in the tunnel regime? To answer it we analysed systematically those contact geometries where at least two channels contribute significantly to the transport properties in the conductance region $G<0.5 G_{0}$. Some representative examples are shown in figure 6. We found that these geometries can be grouped into two categories. In the first one, the electrodes contain several neighbouring atoms that are at similar distances to the atoms in the other electrode, see figure 6(b). At room temperature, practically all the geometries with two channels in the tunnel regime belong to this kind. On the other hand, at $4 \mathrm{~K}$ we also find geometries where the contact breaks at several points giving rise to parallel junctions, see figure 6(a). In both types of geometries the two channels originate from parallel paths with the peculiarity that in the second kind these paths are basically independent, i.e. they do not interfere. Interestingly, we find that the geometries with several parallel junctions are also responsible for the peak at $2 G_{0}$ that appears in the low temperature conductance histogram.

In summary, our low temperature theoretical results are in very good agreement with cryogenic experiments performed in clean conditions and our room temperature results supports the idea of several channels contributing below $1 G_{0}$. However, our theoretical data are not able to reproduce our room temperature experimental findings related to the presence of minima in the variance of the shot noise and Fano factor correlated with the minima in 

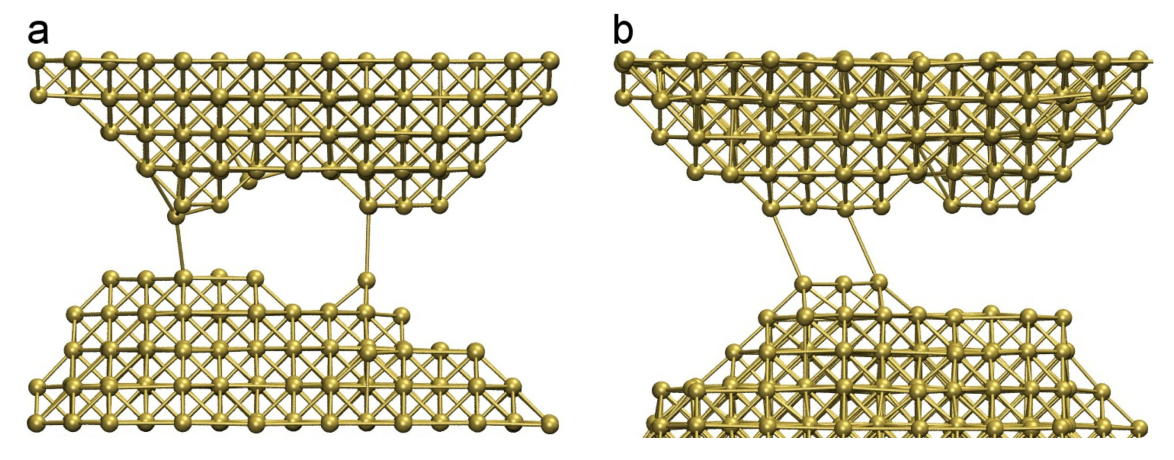

FIG. 6. Two representative examples of contact geometries where two conduction channels give a significant contribution in the tunnel regime $\left(G<0.5 G_{0}\right)$. The total conductance and the individual transmission coefficients in the different examples are (where we consider only channels with $\tau_{i}>0.01$ ): (a) $G=0.29 G_{0}, \tau_{1}=0.28, \tau_{2}=0.11, \tau_{3}=0.01$ and (b) $G=0.15 G_{0}, \tau_{1}=0.09$, $\tau_{2}=0.05$, and $\tau_{3}=0.01$.

their averaged values. The peaks in the experimental conductance histograms, particularly those above $1 G_{0}$, are also considerably more robust than what is seen in the simulations. The survival of higher quantization peaks at room temperature has been observed in many experiments [2, 10, 60, 61].

\section{FURTHER DISCUSSION AND CONCLUSIONS}

As discussed in the previous section, our theoretical simulations have difficulties to reproduce the observed shot noise variance minima, and the robust quantization peaks and noise minima at $G>G_{0}$ at room temperature. The origins of these discrepancies are not clear at the moment. One possibility might be related to the unavoidable limitations in our MD simulations. In particular, one has to bear in mind that the stretching velocity in these simulations is many orders of magnitudes higher than in the experiments. Moreover, because of computational reasons we have to constrain ourselves to relatively small contacts. Although we have investigated these issues systematically within our capabilities and have not found any significant difference in our results upon changes in the stretching velocity, we cannot rule out that this discrepancy is due to systematic deficiencies in our simulations. However, it is worth remarking that our low temperature simulations are in very good agreement with published data in UHV conditions [13] and similar simulations have been very successful 
in reproducing, for instance, the thermopower of different atomic wires [48] or the channel distribution of $\mathrm{Al}$ atomic-size contacts at very low temperatures [27].

Another possibility for this discrepancy might be the fact that these experiments are conducted in air. Though the related environmental parameters such as pressure, temperature or humidity should be relatively stable at each set of measurements, contamination from adsorbates (water molecules for instance) cannot be ruled out. Molecules randomly attached to the junction can surely alter the dynamics and stability of the junctions and the conductance as well, especially in the last stages of the breaking process. Both hydrogen [62, 63] and oxygen [64] are known to incorporate into junctions and alter the available stable atomic configurations. This possibility can be tested by further room temperature experiments in a UHV environment. On the other hand, the disorder of gold junctions may also be relevant [10], which is proven to have effect on peak positions in conductance histogram. Dislocations and other defects introduced by "work hardening" can in principle affect atomic mobility and therefore structural stability, physics very difficult to capture in computationally tractable simulations.

To summarize, we have reported measurements of the shot noise's distribution over ensembles on STM-style gold break junctions at room temperature. The details of the partial noise suppressions seen in the ensemble average results are revealed, showing that outliers can obscure a subensemble with much greater noise suppression compatible with fully transmitting channels. We find signs of non-negligible conduction from additional quantum channels in the nominal tunneling region, and observe minima in the shot noise variance coincident with conductance histogram peaks and averaged shot noise suppressions. Simulations combining MD, a tight-binding model and Green's functions techniques have been conducted. The simulations successfully reproduce many key features of the data and confirm a second channel's participation below $1 G_{0}$. Other aspects of the data, such as the minima in the noise variance near the preferred conductance values and robust conductance histogram peaks above $1 G_{0}$, are not seen in the simulations. One possible explanation is that the simulations are restricted to comparatively small contact geometries and might therefore miss some junction configurations possible in the experiments. Another factor responsible for these discrepancies may be surface contamination in the experiments, altering the breaking dynamics, an effect that is not taken into account in the simulations. Adsorbed contaminants can hinder diffusion and alter the breaking dynamics, effects not simulated 
here. Structural disorder, leading to modifications in the preferred conductances as well as potentially hindering atomic rearrangements, may also be relevant. Further experiments in UHV and under various electrode annealing conditions as well as simulations with yet larger atomic contacts may be able to resolve the relative contributions of these candidate mechanisms.

\section{ACKNOWLEDGMENTS}

D.N. and R.C. acknowledge support of NSF awards DMR-0855607 and DMR-1305879, as well as helpful discussions with P. J. Wheeler. F.P. acknowledges the funding from the Carl Zeiss foundation and the collaborative research center SFB 767. J.C.C. acknowledges financial support from the Spanish MICINN (Contract No. FIS2011-28851-C02-01) and thanks Ran Vardimon, Oren Tal, and Nicolás Agraït for useful discussions. The authors thank the NIC for computer time. 
[1] N. Agraït, A. L. Yeyati, and J. M. van Ruitenbeek, Phys. Rep. 377, 81 (2003).

[2] C. J. Muller, J. M. Krans, T. N. Todorov, and M. A. Reed, Phys. Rev. B 53, 1022 (1996).

[3] E. Scheer, P. Joyez, D. Esteve, C. Urbina, and M. H. Devoret, Phys. Rev. Lett. 78, 3535 (1997).

[4] N. Agraït, C. Untiedt, G. Rubio-Bollinger, and S. Vieira, Phys. Rev. Lett. 88, 216803 (2002).

[5] M. Kumar, R. Avriller, A. L. Yeyati, and J. M. van Ruitenbeek, Phys. Rev. Lett. 108, 146602 (2012).

[6] R. Avriller and T. Frederiksen, Phys. Rev. B 86, 155411 (2012).

[7] Z. Wu, S. Wu, S. Oberholzer, M. Steinacher, M. Calame, and C. Schönenberger, Phys. Rev. B 78, 235421 (2008).

[8] W. Lee, K. Kim, W. Jeong, L. A. Zotti, F. Pauly, J. C. Cuevas, and P. Reddy, Nature 498, 209 (2013).

[9] K. Itakura, K. Yuki, S. Kurokawa, H. Yasuda, and A. Sakai, Phys. Rev. B 60, 11163 (1999).

[10] I. K. Yanson, O. I. Shklyarevskii, S. Csonka, H. van Kempen, S. Speller, A. I. Yanson, and J. M. van Ruitenbeek, Phys. Rev. Lett. 95, 256806 (2005).

[11] L. Venkataraman, J. E. Klare, I. W. Tam, C. Nuckolls, M. S. Hybertsen, and M. L. Steigerwald, Nano Lett. 6, 458 (2006).

[12] E. Scheer, N. Agraït, J. C. Cuevas, A. L. Yeyati, B. Ludoph, A. Martín-Rodero, G. R. Bollinger, J. M. van Ruitenbeek, and C. Urbina, Nature 394, 154 (1998).

[13] R. Vardimon, M. Klionsky, and O. Tal, Phys. Rev. B 88, 161404 (2013).

[14] S. Y. Quek, M. Kamenetska, M. L. Steigerwald, H. J. Choi, S. G. Louie, M. S. Hybertsen, J. B. Neaton, and L. Venkataraman, Nat. Nanotechnol. 4, 230 (2009).

[15] M. Kamenetska, M. Koentopp, A. C. Whalley, Y. S. Park, M. L. Steigerwald, C. Nuckolls, M. S. Hybertsen, and L. Venkataraman, Phys. Rev. Lett. 102, 126803 (2009).

[16] H. Vazquez, R. Skouta, S. Schneebeli, M. Kamenetska, R. Breslow, L. Venkataraman, and M. S. Hybertsen, Nat. Nanotechnol. 7, 663 (2012).

[17] E. Lörtscher, H. B. Weber, and H. Riel, Phys. Rev. Lett. 98, 176807 (2007).

[18] C. M. Guédon, H. Valkenier, T. Markussen, K. S. Thygesen, J. C. Hummelen, and S. J. van der Molen, Nat. Nanotechnol. 7, 305 (2012). 
[19] P. Darancet, J. R. Widawsky, H. J. Choi, L. Venkataraman, and J. B. Neaton, Nano Lett. 12, $6250(2012)$.

[20] N. Fournier, C. Wagner, C. Weiss, R. Temirov, and F. S. Tautz, Phys. Rev. B 84, 035435 (2011).

[21] A. Halbritter, P. Makk, S. Mackowiak, S. Csonka, M. Wawrzyniak, and J. Martinek, Phys. Rev. Lett. 105, 266805 (2010).

[22] P. Makk, D. Tomaszewski, J. Martinek, Z. Balogh, S. Csonka, M. Wawrzyniak, M. Frei, L. Venkataraman, and A. Halbritter, ACS Nano 6, 3411 (2012).

[23] S. V. Aradhya, M. Frei, A. Halbritter, and L. Venkataraman, ACS Nano 7, 3706 (2013).

[24] M. Dreher, F. Pauly, J. Heurich, J. C. Cuevas, E. Scheer, and P. Nielaba, Phys. Rev. B 72, $075435(2005)$.

[25] F. Pauly, M. Dreher, J. K. Viljas, M. Häfner, J. C. Cuevas, and P. Nielaba, Phys. Rev. B 74, $235106(2006)$.

[26] P. Makk, D. Visontai, L. Oroszlány, D. Z. Manrique, S. Csonka, J. Cserti, C. Lambert, and A. Halbritter, Phys. Rev. Lett. 107, 276801 (2011).

[27] C. Schirm, M. Matt, F. Pauly, J. C. Cuevas, P. Nielaba, and E. Scheer, Nat. Nanotechnol. 8, $645(2013)$.

[28] P. Wheeler, J. Russom, K. Evans, N. King, and D. Natelson, Nano Lett. 10, 1287 (2010).

[29] R. Chen, P. J. Wheeler, and D. Natelson, Phys. Rev. B 85, 235455 (2012).

[30] R. Chen, P. J. Wheeler, M. Di Ventra, and D. Natelson, Sci. Rep. 4, 4221 (2014).

[31] W. Schottky, Ann. Phys. 57, 541 (1918).

[32] Y. Blanter and M. Büttiker, Phys. Rep. 336, 1 (2000).

[33] J. B. Johnson, Phys. Rev. 32, 97 (1928).

[34] H. Nyquist, Phys. Rev. 32, 110 (1928).

[35] R. de Picciotto, M. Reznikov, M. Heiblum, V. Umansky, G. Bunin, and D. Mahalu, Nature 389, 162 (1997).

[36] L. Saminadayar, D. C. Glattli, Y. Jin, and B. Etienne, Phys. Rev. Lett. 79, 2526 (1997).

[37] S. Datta, Electronic Transport in Mesoscopic Systems (Cambridge University Press, Cambridge, 1995).

[38] M. Di Ventra, Electrical Transport in Nanoscale Systems (Cambridge University Press, Cambridge, 2008). 
[39] J. C. Cuevas and E. Scheer, Molecular Electronics: An Introduction to Theory and Experiment (World Scientific, Singapore, 2010).

[40] V. Khlus, Sov. Phys. JETP 66, 1243 (1987).

[41] G. B. Lesovik, Sov. Phys. JETP Lett. 49, 592 (1989).

[42] R. Landauer and T. Martin, Physica B 175, 167 (1991).

[43] T. Martin and R. Landauer, Phys. Rev. B 45, 1742 (1992).

[44] M. Büttiker, Phys. Rev. B 46, 12485 (1992).

[45] B. Ludoph and J. M. van Ruitenbeek, Phys. Rev. B 61, 2273 (2000).

[46] B. Ludoph, M. H. Devoret, D. Esteve, C. Urbina, and J. M. van Ruitenbeek, Phys. Rev. Lett. 82, 1530 (1999).

[47] H. E. van den Brom and J. M. van Ruitenbeek, Phys. Rev. Lett. 82, 1526 (1999).

[48] F. Pauly, J. K. Viljas, M. Bürkle, M. Dreher, P. Nielaba, and J. C. Cuevas, Phys. Rev. B 84, $195420(2011)$.

[49] S. Plimpton, J. Comput. Phys. 117, 1 (1995).

[50] http://lammps.sandia.gov.

[51] H. W. Sheng, M. J. Kramer, A. Cadien, T. Fujita, and M. W. Chen, Phys. Rev. B 83, 134118 (2011).

[52] D. Frenkel and B. Smit, Understanding molecular simulation (Academic Press, San Diego, 2004).

[53] F. Pauly, J. Viljas, U. Huniar, M. Häfner, S. Wohlthat, M. Bürkle, J. C. Cuevas, and G. Schön, New J. Phys. 10, 125019 (2008).

[54] M. J. Mehl and D. A. Papaconstantopoulos, Computational Materials Science (edited by C. Fong) (World Scientific, Singapore, 1998).

[55] F. Guinea, C. Tejedor, F. Flores, and E. Louis, Phys. Rev. B 28, 4397 (1983).

[56] M. Brandbyge, N. Kobayashi, and M. Tsukada, Phys. Rev. B 60, 17064 (1999).

[57] H. Ohnishi, Y. Kondo, and K. Takayanagi, Nature 395, 780 (1998).

[58] A. I. Yanson, G. R. Bollinger, H. E. van den Brom, N. Agraït, and J. M. van Ruitenbeek, Nature 395, 783 (1998).

[59] R. Vardimon and O. Tal, private communication (2014).

[60] Z. Gai, Y. He, H. Yu, and W. S. Yang, Phys. Rev. B 53, 1042 (1996).

[61] J. L. Costa-Krämer, Phys. Rev. B 55, R4875 (1997). 
[62] S. Csonka, A. Halbritter, G. Mihály, E. Jurdik, O. I. Shklyarevskii, S. Speller, and H. van Kempen, Phys. Rev. Lett. 90, 116803 (2003).

[63] R. N. Barnett, H. Häkkinen, A. G. Scherbakov, and U. Landman, Nano Letters 4, 1845 (2004).

[64] W. H. A. Thijssen, M. Strange, J. M. J. aan de Brugh, and J. M. van Ruitenbeek, New J. of Phys. 10, 033005 (2008). 